Ruhr-Universität Bochum

PD Dr. med. Stefan Georg Schröder

Dienstort: KMG Klinikum Güstrow

Abteilung für Psychiatrie u. Psychotherapie

\title{
Leben Demenzkranke zu Hause länger als im Heim?
}

\author{
Kumulative \\ Inaugural-Dissertation \\ zur \\ Erlangung des Doktorgrades der Medizin \\ einer \\ Hohen Medizinischen Fakultät \\ der Ruhr-Universität Bochum
}

vorgelegt von

Daniel Lankers

aus Bonn-Duisdorf

2010 
Dekan: $\quad$ Prof. Dr. med. Gert Muhr

Referent: $\quad$ PD Dr. med. Stefan G. Schröder

Koreferent: Prof. Dr. med. Ludger Pientka

Tag der Mündlichen Prüfung: 18.10.2011 


\section{Abstract}

Lankers

Daniel

Leben Demenzkranke zu Hause länger als im Heim?

\section{Problem:}

Wie eine Vielzahl von Studien zeigt, nimmt die Zahl der Neuerkrankungen an Demenz in unserer Gesellschaft jährlich zu. In diesem Zusammenhang stehen zwei gesellschaftliche Aufgaben im Vordergrund: die Sicherstellung der adäquaten Betreuung von an Demenz Erkrankten auch in Zukunft sowie die Deckung der Kosten, die dadurch verursacht werden. Dabei hat die Überlebenszeit von Demenzkranken - gerade auch für gesundheitspolitische Planungen - eine zentrale Bedeutung. Deshalb lautet die Fragestellung unserer über 8 Jahre angelegten Follow-up-Studie: Beeinflusst die Wohnform die Überlebenszeit Demenzkranker?

\section{Methode:}

Von 173 Patienten mit Alzheimer- oder vaskulärer Demenz (ICD-10-Nr. F00 und F01) wurden klinische und soziale Daten erfasst. Bei verstorbenen Patienten wurde nach Befragung der Angehörigen das exakte Todesdatum ermittelt. Anschließend wurden die Überlebenszeiten mit der entsprechenden Wohnform korreliert. Unter Anwendung des CoxModells wurden Sterberisiken ermittelt. Der Bestimmung der medianen Überlebenszeit in Abhängigkeit von der Wohnform diente die Überlebenszeitanalyse nach Kaplan-Meier.

\section{Ergebnis:}

Die Untersuchung zeigt, dass ein deutlicher Unterschied in der Überlebenszeit Demenzkranker besteht, je nachdem, ob sie im Seniorenheim oder zu Hause gepflegt wurden. Adjustiert nach Alter und kognitiver Leistungsminderung hatten Patienten im Seniorenheim ein um 53,1\% höheres relatives Sterberisiko (Hazard Ratio) als zu Hause Betreute $(p=0,047)$.

\section{Diskussion:}

Unserer Studie nach zeigen zu Hause gepflegte Patienten unter Alters-, Geschlechts- und Schweregradadjustierung ein geringeres relatives Sterberisiko (Hazard Ratio) als im Seniorenheim Betreute. Auch leben demenzkranke Frauen länger als demenzkranke Männer.

Die Abschätzung der Überlebenszeiten von Demenzkranken hat unseres Erachtens u. a. die praktische Bedeutung, Angehörige bezüglich der Prognose fundierter beraten zu können, damit diese besser ihre eigenen Grenzen erkennen und sich entsprechend für eine häusliche oder institutionelle pflegerische Versorgung entscheiden können. Dies hätte im Sinne einer psychiatrischen Primärprävention eine Verbesserung des Gesundheitszustandes der pflegenden Angehörigen zur Folge, da diese eine Hochrisikopopulation für psychiatrische Morbidität darstellen.

Nach unserer Erkenntnis wurden bislang nur wenige Studien zur Frage der Versorgungsqualität von Patienten mit Demenz in Abhängigkeit von der gewählten Wohnform durchgeführt. Keine davon untersuchte den Einfluss der Wohnform auf die Mortalität. Unsere Ergebnisse zeigen jedoch gerade hier signifikante Unterschiede und weisen auf einen noch zu wenig beachteten Bereich der Versorgungsforschung hin. 
Inhaltsverzeichnis

$\begin{array}{ll}\text { Verzeichnis der Tabellen } & \text { Seite } 5\end{array}$

$\begin{array}{ll}\text { Verzeichnis der Abbildungen } & \text { Seite } 6\end{array}$

$\begin{array}{ll}\text { Einleitung } & \text { Seite } 7\end{array}$

$\begin{array}{ll}\text { Zielsetzung } & \text { Seite } 9\end{array}$

$\begin{array}{ll}\text { Methode } & \text { Seite } 10\end{array}$

$\begin{array}{ll}\text { Ergebnis } & \text { Seite } 12\end{array}$

$\begin{array}{ll}\text { Diskussion } & \text { Seite } 13\end{array}$

$\begin{array}{ll}\text { Literaturverzeichnis } & \text { Seite } 16\end{array}$

$\begin{array}{ll}\text { Anhang } & \text { Seite } 19\end{array}$

Danksagung $\quad$ Seite 22

$\begin{array}{ll}\text { Lebenslauf } & \text { Seite } 23\end{array}$ 
Verzeichnis der Tabellen

T1 Beschreibung der Kohorte

T2 Mediane Überlebenszeiten

T3 Variablen der Cox-Regression 
Verzeichnis der Abbildungen

A1 Schweregrad der Demenz nach Wohnform (gemäß MMST $\leq 20$ bzw. $\leq 10$ )

A2 Überlebenszeit nach Wohnform 


\section{Einleitung}

Zurzeit leben in Deutschland etwa 82 Millionen Menschen, bis zum Jahr 2050 wird sich diese Zahl nach Angaben des Statistischen Bundesamtes Deutschland um etwa neun Prozent auf knapp 75 Millionen Einwohner verringern [23]. Gleichzeitig schreitet das Altern der Bevölkerung kontinuierlich fort. Der Bevölkerungsanteil derjenigen im Erwerbsalter wird voraussichtlich überproportional um 20 Prozent schrumpfen, während die Anzahl der über 65-Jährigen und der über 80-Jährigen um 54 Prozent bzw. 174 Prozent zunehmen wird [3]. Diese Verschiebung der Altersstruktur liegt einerseits an der sinkenden Geburtenrate, andererseits an der steigenden Lebenserwartung und der Tatsache, dass insbesondere die Sterblichkeit im hohen Lebensalter weiter sinkt [24]. Durch die zunehmende Hochaltrigkeit entsteht die wachsende Personengruppe des so genannten „4.

Lebensalters“. Somit steigt die Wahrscheinlichkeit, dass mehr alt gewordene Menschen nicht-tödliche (chronische) Krankheitsereignisse erleiden [17]. Insbesondere Demenzerkrankungen sind zu einem großen Gesundheitsproblem geworden, da diese wie keine andere Krankheitsgruppe die Entstehung von Pflegebedürftigkeit und den Bedarf an Plätzen in stationären Pflegeeinrichtungen zu bestimmen scheinen. Zwei Drittel des Krankenbestandes und 60\% der Neuerkrankungen entfallen auf die über 80Jährigen, nur drei Prozent hingegen auf die unter 65-Jährigen [1]. Im Jahr 2000 schätzte Bickel die Zahl der Demenzkranken auf 950.000. Jährlich ist mit 192.000 bis 227.000 neuen Fällen zu rechnen.

In Bezug auf die Krankheit Demenz stehen zwei gesellschaftliche Aufgabenbereiche im Vordergrund: die Deckung der Kosten, die durch Demente verursacht werden und die Sicherstellung ihrer adäquaten Betreuung. Die weltweiten direkten Kosten der Demenz im Jahr 2003 werden auf 156 Milliarden US-Dollar geschätzt, bei einer Prävalenz von 27,7 Millionen dementen Personen. 92\% der Kosten entstehen in den Industrienationen, deren Demente 38\% der weltweiten Prävalenz ausmachen [26]. In Deutschland werden die Gesamtkosten der Pflege aus 
unterschiedlichen Quellen finanziert: Einerseits durch die Mittel der Pflegeversicherung, welche je nach Pflegestufe zwischen 51,1 und 54,5 Prozent der anfallenden Kosten übernimmt, andererseits von der versorgten Person selbst, sei es durch die eigene Rente, private Vermögenswerte oder die Zahlungen naher Angehöriger [6]. Im Jahr 2005 gab es in Deutschland 9.105 vollstationäre Alteneinrichtungen mit insgesamt ca. 749.000 Plätzen. $55 \%$ dieser Einrichtungen wurden von freien, gemeinnützigen Organisationen, 37\% von privaten, kommerziellen Organisationen und 8,0\% vom Staat finanziert [14]. Etwa 60\% der Demenzkranken in Deutschland leben in Privathaushalten und werden zu rund 92\% von Familienangehörigen versorgt [3].

Entsprechend muss ein großer Teil der Leistungen von den Angehörigen getragen werden. Hierzu zählen nicht nur die indirekten Kosten (z. B. geleistete Betreuungszeit), auch die Zuzahlung zu Medikamenten oder der Eigenanteil, der für die Unterkunft/Verpflegung des Erkrankten aufzubringen ist, bedeutet für die betroffenen Angehörigen eine große Belastung [10]. Auch wird sich in den kommenden Jahren die Versorgung Demenzkranker durch Angehörige zunehmend schwierig gestalten: Über $60 \%$ der Personen, die als Hauptpflegende Betreuung in häuslichen Pflegearrangements übernehmen, sind über 55 Jahre alt und können in Zukunft die Pflegeverantwortung nicht mehr selbstverständlich übernehmen, da sie dann u. U. selbst nicht mehr in der Lage sind, körperlich belastende Pflegetätigkeiten zu leisten. Zudem steigt bei Frauen, die mit $80 \%$ den Großteil der Pflegenden darstellen, die Zahl der Berufstätigen [21]. 


\section{Zielsetzung}

Die Altenpflege steht damit vor einer Herausforderung: Wachsende Betreuungsaufgaben werden bei verminderten familiären Ressourcen zu bewältigen sein. In diesem Zusammenhang hat die Überlebenszeit von Demenzkranken - gerade auch für gesundheitspolitische Planungen - eine zentrale Bedeutung. Nach überwiegender Meinung beträgt die mittlere Überlebenszeit bei Altersdemenz, von den ersten klinischen Symptomen an gerechnet, etwa zehn Jahre [1, 2, 9]. Da psychosoziale Einflussfaktoren auf die Mortalität bei Demenz bislang noch zu wenig erforscht sind, wurde für die vorliegende Studie der Einfluss der Wohnform auf die Überlebenszeit von 173 Patienten untersucht. Gibt es diesbezüglich Unterschiede zwischen der häuslichen Pflege und der Unterbringung im Seniorenheim? 


\section{Methode}

Überlebenszeiten werden normalerweise ab Krankheitsbeginn berechnet, Demenzerkrankungen beginnen jedoch zumeist schleichend und werden anfangs regelhaft übersehen. Das Zeitintervall ab Symptombeginn Jahre später zu rekonstruieren, stellt sich daher oft als schwierig heraus. Der Termin der Erstdiagnose variiert zwar stark, je nach Aufmerksamkeit des konsultierten Arztes und Informationsstand der Angehörigen, aber auch abhängig von Persönlichkeitsvariablen der Betroffenen, bildet aber trotzdem einen klar definierten und fixen Startpunkt für Betrachtungen zur Überlebenszeit. Angesichts der Progredienz der Erkrankung ist eine spätere Erstdiagnose an einem niedrigeren MMST-Punktwert leicht ablesbar. Daher wurden die Überlebenszeiten ab dem Zeitpunkt der Erstvorstellung in der Institutsambulanz des Westfälischen Zentrums Bochum (Universitätsklinik) berechnet.

In die Studie wurden Patienten mit den Diagnosen Alzheimer-Demenz (AD) und vaskuläre Demenz (VD) einbezogen. Als Einschlusskriterium galten dementsprechend die ICD-10-Nummern F00 und F01. Im Zeitraum zwischen 1995 und 2001 hatten 173 Demenzkranke mit AD und VD die Institutsambulanz des Westfälischen Zentrums Bochum (Universitätsklinik) zur weiteren Diagnostik aufgesucht. Bei allen Patienten wurde bei der Erstuntersuchung der Mini-Mental-Status-Test (MMST) durchgeführt, außerdem wurden soziale Faktoren, wie die Wohnform, zum Zeitpunkt des Erstkontaktes festgehalten. Im Rahmen des Follow-up wurde der Überlebensstatus der Patienten telefonisch ermittelt. Im Mittel erfolgte diese Nachfrage 7,98 Jahre nach Erstkontakt. Bei bereits verstorbenen Personen wurde die Überlebenszeit nach Erstkontakt berechnet (Tab. 1).

Bei 24 Patienten konnte das Überleben nach Erstvorstellung nicht ermittelt werden. Bei 4 weiteren Patienten war kein MMST durchgeführt worden, sodass in die Berechnung der Überlebenszeiten 145 Personen eingingen. Signifikante Unterschiede zwischen den Gruppen wurden für den MMST und 
das Alter bei Erstvorstellung (t-Test für die Mittelwertgleichheit), die Überlebenszeit nach Erstvorstellung (Log-Rang-Test) und die Geschlechterverteilung ( $X^{2}$-Test nach Pearson) bestimmt. Die Patientendaten wurden nach den Wohnformen „im Seniorenheim lebend“ und „zu Hause lebend“ ausgewertet.

Für alle statistischen Verfahren wurde ein $p$-Wert $<0,05$ als statistisch signifikant festgelegt. Die statistische Bearbeitung der Daten erfolgte mit dem Programm SPSS 15.0 für Windows. Die Analyse des relativen Sterberisikos wurde anhand des Cox-Regressionsmodells [5] unter Berücksichtigung der Kofaktoren Alter, Geschlecht, MMST und Diagnose (AD vs. VD) durchgeführt. Der Bestimmung der medianen Überlebenszeit in Abhängigkeit von der Wohnform diente die Überlebenszeitanalyse nach Kaplan-Meier. 


\section{Ergebnis}

Zwischen der Gesamtkohorte und der Gruppe von Personen mit unvollständiger Datenlage ergaben sich weder nach den uns vorliegenden soziodemographischen und klinischen Variablen noch nach den statistischen Auswertungen signifikante Unterschiede. Eine Ausnahme bildete die mediane Überlebenszeit nach Erstvorstellung, da der eruierbare Wert in der Gruppe der Patienten mit unvollständiger Datenlage auf lediglich 4 von 28 Patienten beruhte.

Zum Zeitpunkt der Datenerfassung (Juni 2006) waren 117 der 145 Patienten mit vollständiger Datenlage verstorben. Die 48 im Seniorenheim lebenden Patienten waren bei Erstkontakt etwa 7,1 Jahre älter als die 97 häuslich Gepflegten $(p<0,001)$. Das durchschnittliche Ergebnis des MMST war um 4,2 Punkte niedriger als das der zu Hause Lebenden ( $p<0,001 ;$ Abb. 1). Im Seniorenheim lebten 42 Frauen (87,5\%), zu Hause 64 (66\%), p=0,006. Die Berechnungen nach Kaplan-Meier ergaben eine mediane Überlebenszeit der Patienten im Seniorenheim von 29,2 Monaten (2,43 Jahre) im Gegensatz zur Überlebenszeit der zu Hause Lebenden mit 55,5 Monaten (4,63 Jahre; $p=0,043 ;$ Abb. 2, Tab. 2).

Die Adjustierung des Cox-Regressionsmodells an den Variablen MMSTWert, Alter, Geschlecht und Diagnose (AD vs. VD) erbrachte die in Tab. 3 dargestellten Werte. Die Berechnung der Hazard Ratio nach Cox im Vergleich zwischen den Wohnformen ergab den Wert 1,531 (95\%-Konfidenzintervall: 1,005-2,333), $p=0,047$. Aus diesem Ergebnis lässt sich ablesen, dass das relative Sterberisiko von Demenzpatienten im Seniorenheim - adjustiert an den oben genannten Variablen - um 53,1\% erhöht ist. 


\section{Diskussion}

Unsere Untersuchung zeigt, dass ein deutlicher Unterschied in der Überlebenszeit an Demenz Erkrankter besteht, je nachdem, ob sie im Seniorenheim oder zu Hause gepflegt wurden. Zu Hause versorgte Demenzpatienten haben nach unserer Studie ein um 53,1\% niedrigeres relatives Sterberisiko als die Patienten im Seniorenheim. Einerseits ergeben die Berechnungen nach Kaplan-Meier bei der Gruppe der zu Hause lebenden Patienten ein um 26,3 Monate (2,2 Jahre) längeres medianes Überleben. Andererseits bleibt bei dieser Betrachtung unberücksichtigt, dass die Gruppe der im Seniorenheim Lebenden bereits zu Anfang des Betrachtungszeitraumes ein um 7,1 Jahre höheres Alter und, dem um durchschnittlich 4,2 Punkte schlechteren MMST-Wert entsprechend, eine niedrigere kognitive Fähigkeit durch die im Mittel schon weiter fortgeschrittene Demenz aufweist. Berücksichtigt man diesen Sachverhalt ebenso wie die unterschiedliche Geschlechtsverteilung, ergibt sich in der Regressionsberechnung nach dem Cox-Modell (Hazard Ratio) die niedrigere Sterbewahrscheinlichkeit der häuslich Versorgten. Dies ließe in einer prospektiv angelegten Studie mit einheitlichen Patientengruppen eine deutlich höhere Überlebenszeit für zu Hause versorgte Patienten erwarten.

Der erhebliche Unterschied der Überlebenszeiten zwischen den Patienten mit vollständiger und unvollständiger Datenlage lässt sich nach einer Reevaluation der Einzelfälle darauf zurückführen, dass in der kleinen Gruppe der Patienten $(n=4)$, bei denen keine psychometrische Untersuchung durchgeführt, wohl aber die Überlebenszeit bestimmt wurde, bereits ein sehr fortgeschrittenes Demenzstadium vorlag. Ein - wenn auch geringer - Bias könnte auf dieser Selektion beruhen. Die ermittelten medianen Überlebenszeiten müssten somit allenfalls geringfügig nach unten korrigiert werden. In dieser Studie wird zwischen Alzheimer- und vaskulärer Demenz unterschieden, obwohl neuere Untersuchungen eine große Überschneidung dieser beiden nosologischen Entitäten auf histopathologischer [18] und klinischer [22] Ebene zeigen. Der Einfluss der Demenzätiologie auf die 
Sterblichkeit wird uneinheitlich bewertet: Je nach Studie hat die AD eine etwas günstigere Prognose als die VD [16] oder beide Hauptdemenzformen unterscheiden sich nicht wesentlich bezüglich der Überlebenszeit [9]. Die unterschiedliche Geschlechterverteilung wurde zwar in der CoxRegression berücksichtigt - so zeigen Frauen unserer Studie zufolge eine ca. 2,5-fach erhöhte Überlebenswahrscheinlichkeit gegenüber Männern -, allerdings wird auch der Einfluss des Geschlechts auf die Sterblichkeit von Demenzkranken kontrovers beurteilt, ohne dass es diesbezüglich einen wissenschaftlichen Konsens gibt [9].

Aufgrund des retrospektiven Designs der Studie konnten keine Nebendiagnosen erfasst werden. Auch wenn es Hinweise darauf gibt, dass die Mortalität der Erkrankten hauptsächlich vom Alter und Schweregrad der Demenz und weniger von weiteren gravierenden Erkrankungen (wie z. B. Herz-Kreislauf- oder Krebserkrankungen) abhängt [2], sollten diese somatischen Diagnosen in künftigen Studien erfasst werden. Das gilt auch für weitere wichtige Einflussfaktoren wie die Medikation (z. B. bezüglich Sturzgefährdung) oder die Art der Betreuung der zu Hause versorgten Patienten (durch Lebenspartner, Kindergeneration, ambulanten Pflegedienst).

Die Abschätzung der Überlebenszeiten von Demenzkranken hat unseres Erachtens u. a. die praktische Bedeutung, Angehörige bezüglich der Prognose fundierter beraten zu können, damit diese besser ihre eigenen Grenzen erkennen und sich entsprechend für eine häusliche oder institutionelle pflegerische Versorgung entscheiden können [12]. Dies hätte im Sinne einer psychiatrischen Primärprävention eine Verbesserung des Gesundheitszustandes der pflegenden Angehörigen zur Folge, da diese eine Hochrisikopopulation für psychiatrische Morbidität darstellen. Studien zur häuslichen Pflege von Demenzkranken konstatieren eine signifikant höhere Belastung der pflegenden Angehörigen im Vergleich zu Pflegenden nicht an Demenz Leidender [4]. Ihren eigenen Angaben zufolge sind 42\% der Angehörigen schwer, 41\% sogar sehr schwer belastet [14]. Angehörige, die sich intensiv um einen Pflegebedürftigen kümmerten, litten häufiger an 
körperlichen Beschwerden wie Erschöpfung, Gliederschmerzen, Herz- und Magenproblemen als die Normalbevölkerung [8]. Unter anderem aus diesen Gründen gilt Demenz als Hauptrisikofaktor für eine Heimunterbringung [25]. Durch die professionelle Versorgung kann im Seniorenheim besonders auf somatische Probleme meist besser eingegangen werden [11]. So stellen Mangelernährung und Dehydrierung zentrale Probleme der Pflege von Demenzkranken dar [20], denen im häuslichen Milieu häufig schwerer zu begegnen ist. Der fünfte Altenbericht der Bundesregierung kommt zu dem Ergebnis, dass bei pflegenden Angehörigen das Wissen um die Bedürfnisse der an Demenz leidenden Personen oft nicht ausreichend ist [3]. Auch gibt es Hinweise dafür, dass dem gestörten Schlaf-Wach-Rhythmus von Demenzkranken, der zu Hause eine große Belastung für die Angehörigen darstellt, im Altenheim durch den Schichtdienst besser begegnet werden kann [7]. Für die Heimunterbringung spricht auch, dass nach Jakubaschk die Selbstgefährdung der an Demenz Erkrankten zu Hause höher einzuschätzen ist als im Pflegeheim [15] und Seniorenheime häufig baulich besser an die Anforderungen Pflegebedürftiger angepasst sind [13]. Dementgegen gibt es Hinweise, dass ein inadäquater Einsatz von Psychopharmaka in Seniorenheimen in Deutschland häufig ist, bei einer Verordnungsrate von $55,6 \%$ der Bewohner [19]. Inwieweit sich dies auf die Überlebenszeit Demenzkranker auswirkt, bleibt zu untersuchen. Auch das Thema Lebensqualität hat noch wenig Beachtung erlangt, wobei vermutlich häufig in Pflegeheimen die sozialen Bedürfnisse der Kranken (u. a. zwischenmenschlicher Kontakt und sinnvolle Beschäftigung) weniger befriedigt werden $[11,19]$.

Nach unserer Erkenntnis wurden bislang nur wenige Studien zur Frage der Versorgungsqualität von Patienten mit Demenz in Abhängigkeit von der gewählten Wohnform durchgeführt. Keine davon untersuchte den Einfluss der Wohnform auf die Mortalität. Unsere Ergebnisse zeigen jedoch gerade hier signifikante Unterschiede und weisen auf einen noch zu wenig beachteten Bereich der Versorgungsforschung hin. 


\section{Literaturverzeichnis}

[1] Bickel, H. (2000). Demenzsyndrom und Alzheimer Krankheit: Eine Schätzung des Krankenbestandes und der jährlichen Neuerkrankungen in Deutschland. Gesundheitswesen 62, 211-218

[2] Bonsignore, M., Heun, R. (2003). Mortality in Alzheimer's disease. Dement Geriatr Cogn Disord 15, 231-236

[3] Bundesministerium für Familie, Senioren, Frauen und Jugend (2005). Fünfter Bericht zur Lage der älteren Generation. Eigenverlag, Berlin

[4] Bundesministerium für Familie, Senioren, Frauen und Jugend (2002). Vierter Bericht zur Lage der älteren Generation. Eigenverlag, Berlin

[5] Cox, D. (1972). Regression models and life tables. J R Stat Soc B $34,187-220$

[6] Eurofamcare (2004). National Background report for Germany 2004. http://www.uke.uni-hamburg.de/eurofamcare

[7] Gehrman, P., Marler, M., Martin, JL. et al. (2004). The timing of activity rhythms in patients with dementia is related to survival. J Gerontol A Biol Sci Med Sci 59,1050-1055

[8] Gräßel, E. (1998). Häusliche Pflege demenziell und nicht-demenziell Erkrankter. Teil II: Gesundheit und Belastung der Pflegenden. Z Gerontol Geriatr 31, 57-62

[9] Guehne, U., Riedel-Heller, S., Angermeyer, M.C. (2005). Mortality in dementia. Neuroepidemiology 25, 153-162

[10] Hallauer, J.F., Berger, K., Ruckdäschel, S. (2002). Sozio-ökonomische Aspekte - nationale und internationale Untersuchungsergebnisse. In: Hallauer, J.F., Kurz, A. (Hrsg.). Weißbuch Demenz - Versorgungssituation relevanter Demenzerkrankungen in Deutschland. Thieme, Stuttgart, S. 19-23

[11] Hancock, G.A., Woods, B., Challis, D., Orrell, M. (2006). The needs of older people with dementia in residential care. Int J Geriatr Psychiatry $21,43-49$

[12] Hötte, S.D., Lankers, D., Kissler, S. et al. (2010). MMST zur Überlebensdiagnostik bei Demenz. Psychiat Prax 37 (in Druck)

[13] Infratest Sozialforschung (2003). Hilfe- und Pflegebedürftige in Privathaushalten in Deutschland 2002. Schnellbericht. http://www.tnsinfratest-sofo.com/downloads/mug3sb.pdf 
[14] Infratest Sozialforschung (2006). Hilfe- und Pflegebedürftige in Alteneinrichtungen in Deutschland 2005. Schnellbericht.

http://www.bmfsfj.de/RedaktionBMFSFJ/Abteilung3/Pdf-Anlagen/hilfeund-pflegebeduerftige-in-alteneinrichtungen

[15] Jakubaschk, J., Hunziker, R. (1988). Patienten mit seniler Demenz eine Gruppe der neuen Langzeitpatienten. Psychiatr Prax 15, 115-121

[16] Kissler, S., Hötte, S.D., Lankers, D. et al. (2008). Welchen Einfluss haben zerebrale Durchblutungsstörungen auf die Überlebenszeit Demenzerkrankter? Z Gerontol Geriatr 41, 51-55

[17] Knesebeck, O. vd., Döhner, H., Kaduszkiewicz, H. et al. (2006). Forschung zur Versorgung im höheren Lebensalter. Prävention, Case Management und Versorgung von Demenz. Bundesgesundheitsbl Gesundheitsforsch Gesundheitsschutz 49,167-174

[18] MRC CFAS (2001). Pathological correlates of late-onset dementia in a multicentre, community-based population in England and Wales. Lancet 357, 169-175

[19] Pantel, J., Weber, B., Bockenheimer-Lucius, G., Ebsen, I. (2007). Psychopharmaka im Altenpflegeheim. http://www.bhf-bankstiftung.de/2007/Abschlussbericht.pdf

[20] Rainer, M.K., Mucke, H.A., Masching, A.J. et al. (2005). Zeitgemäßes Management von nicht kognitiven Symptomen bei Demenz. Psychiatr Prax 32, 31-38

[21] Schneekloth, U., Müller, U. (2000). Wirkungen der Pflegeversicherung. Schriftenreihe des Bundesministeriums für Gesundheit. Bd. 127. Nomos, Baden-Baden

[22] Schröder, S.G. (2006). Psychopathologie der Demenz. Symptomatologie und Verlauf dementieller Erkrankungen. Schattauer, Stuttgart

[23] Statistisches Bundesamt Deutschland (2003). Bevölkerung Deutschlands bis 2050 - Ergebnisse der 10. koordinierten Bevölkerungsvorausberechnung. Wiesbaden. Eigenverlag, Berlin

[24] Vaupel, J.W., v. Kistowski, K.G. (2005). Der bemerkenswerte Anstieg der Lebenserwartung und sein Einfluss auf die Medizin. Bundesgesundheitsbl Gesundheitsforsch Gesundheitsschutz 48, 586592

[25] Weyerer, S., Schaufele, M., Schrag, A., Zimber, A. (2004).

Demenzielle Störungen, Verhaltensauffälligkeiten und Versorgung von Klienten in Einrichtungen der Altentagespflege im Vergleich mit 
Heimbewohnern: eine Querschnittsstudie in acht badischen Städten. Psychiatr Prax 31, 339-345

[26] Wimo, A., Jonsson, L., Winblad, B. (2006). An estimate of the worldwide prevalence and direct costs of dementia in 2003. Dement Geriatr Cogn Disord 21, 175-181 


\section{Anhang}

Tabelle 1 (T1)

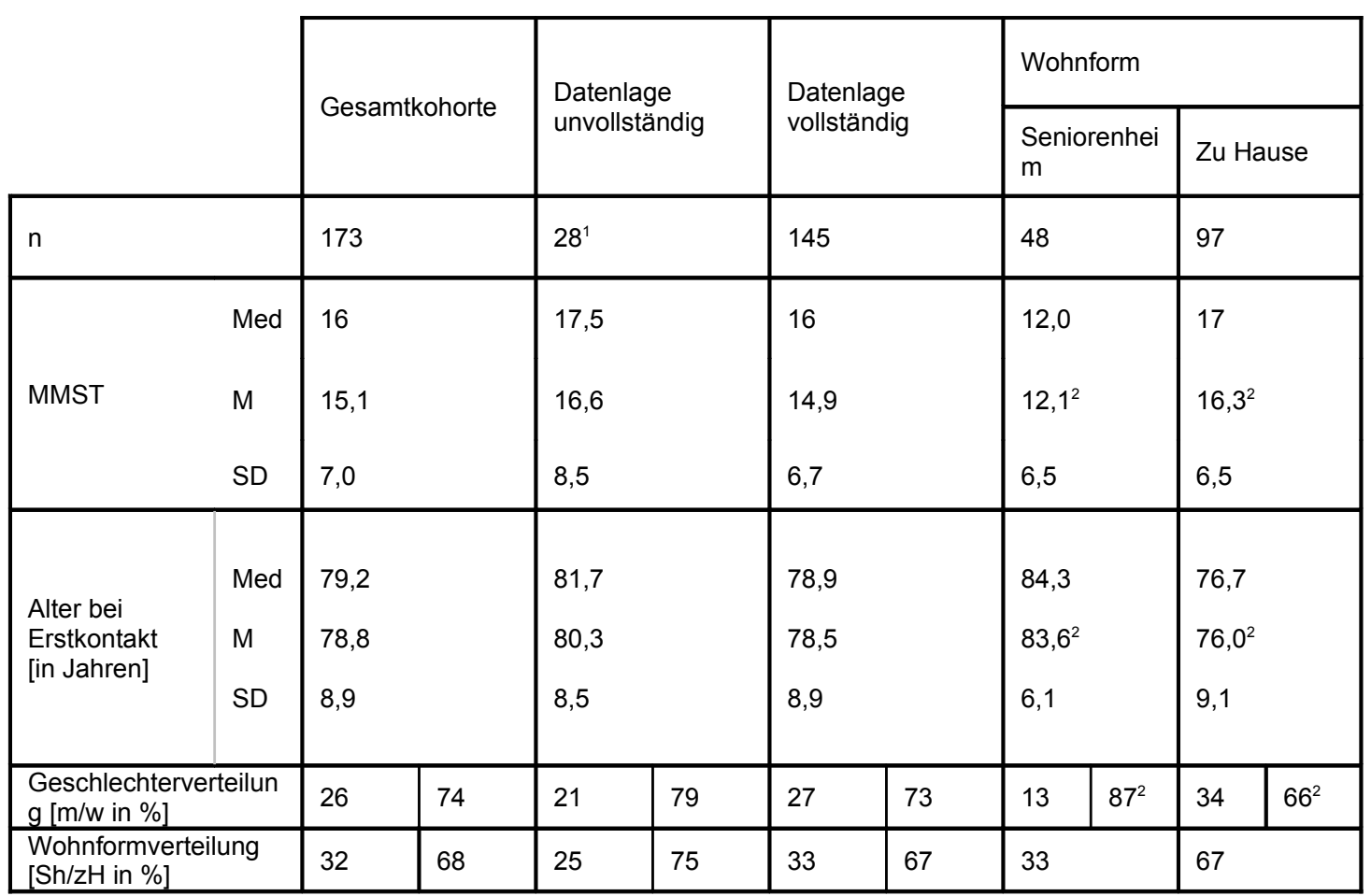

${ }^{1}$ fehlende Überlebenszeit bei 24 Personen, fehlender MMST bei 4 Personen, ${ }^{2}$ signifikante Unterschiede zwischen den Wohnformen (Alter und MMST: t-Test, Geschlecht: ChiQuadrat), n: Anzahl, Med: Median, M: Mittelwert, SD: Standardabweichung, zH: zu Hause lebend, Sh: im Seniorenheim lebend

Tabelle 2 (T2)

\begin{tabular}{|l|l|l|l|l|l|}
\cline { 5 - 6 } & & & & \multicolumn{2}{l|}{ Wohnform } \\
\cline { 5 - 6 } & Gesamtkohorte & $\begin{array}{l}\text { Datenlage } \\
\text { unvollständig }\end{array}$ & $\begin{array}{l}\text { Datenlage } \\
\text { vollständig }\end{array}$ & Seniorenheim & Zu Hause \\
\cline { 5 - 6 } & 173 & $28^{1}$ & 145 & 48 & 97 \\
\hline $\begin{array}{l}\text { Mediane } \\
\text { Überlebenszeit [in } \\
\text { Monaten] }\end{array}$ & 44,0 & $6,7^{2}$ & 44,2 & $29,2^{3}$ & $55,5^{3}$ \\
\hline
\end{tabular}

${ }^{1}$ fehlende Überlebenszeit bei 24 Personen, fehlender MMST bei 4 Personen, ${ }^{2}$ signifikanter Unterschied zur Gesamtkohorte (Log-Rang). Wert beruht auf 4 Personen, ${ }^{3}$ signifikanter Unterschied zwischen den Wohnformen (Log-Rang), n: Anzahl

Tabelle 3 (T3) 


\begin{tabular}{|l|l|l|l|l|}
\cline { 3 - 5 } \multicolumn{1}{c|}{} & \multirow{2}{*}{ Signifikanz } & \multirow{2}{*}{\begin{tabular}{|l|l|} 
Exp(B) \\
\cline { 3 - 4 } \multicolumn{1}{c|}{}
\end{tabular}} & & \multicolumn{2}{c|}{$95.0 \%$ Konfidenzintervall für Exp(B) } \\
\cline { 3 - 5 } & & (hazard ratio) & Untere & Obere \\
\hline Alter & .000 & 1.046 & 1.023 & 1.070 \\
Geschlecht & .000 & 2.583 & 1.678 & 3.978 \\
MMST & .051 & .971 & .942 & 1.000 \\
Diagnose & .212 & .754 & .484 & 1.174 \\
\hline
\end{tabular}




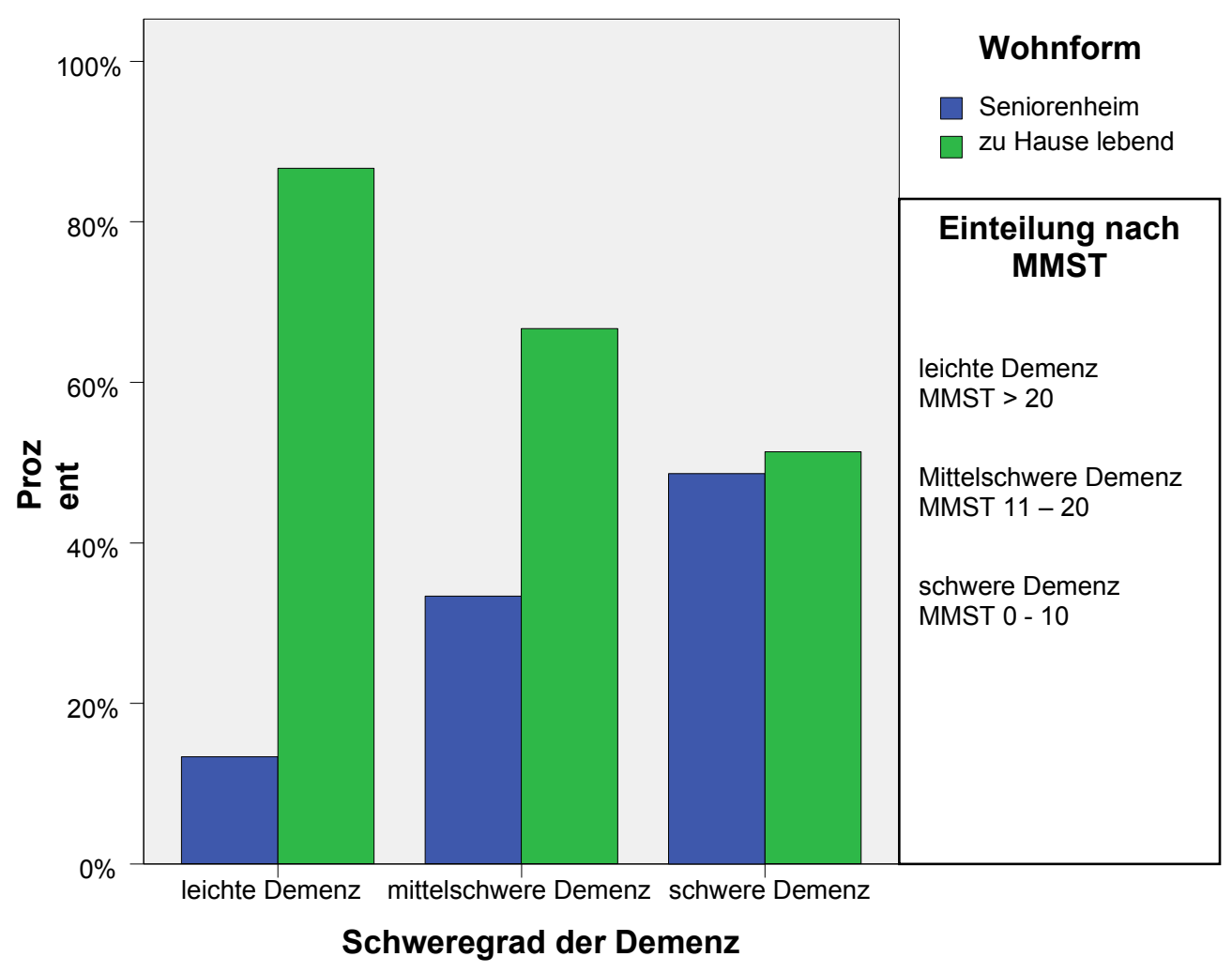

Abbildung 1 (A1)

Überlebenszeit nach Wohnform

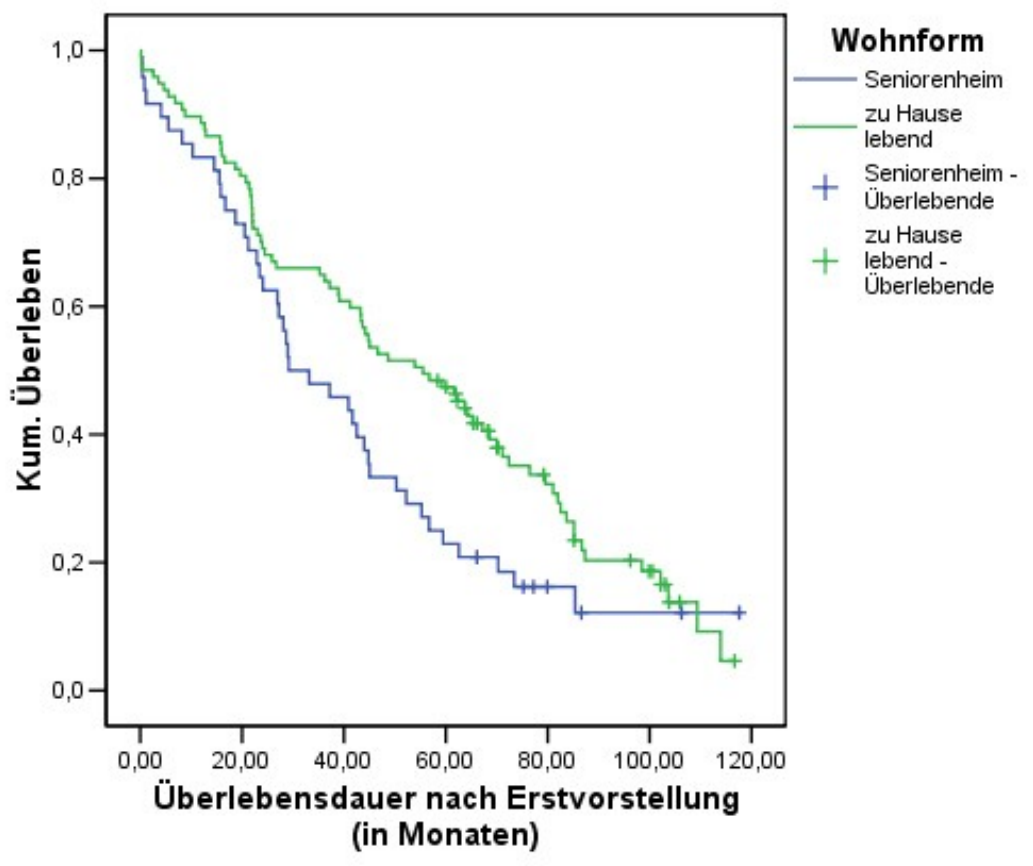

Abbildung 2 (A2) 


\section{Danksagung}

Mein besonderer Dank gebührt meinem Doktorvater Privat-Dozent Dr. Stefan Georg Schröder, für seine freundschaftliche Betreuung, nicht endende Telefonate voller Ideen und Anregungen und sein Durchhaltevermögen. Danke, für immer neue Motivation und Ermutigung.

Meinen Studienkollegen, Sebastian Kissler und Sebastian Hötte, danke ich für ungezählte Tage und Abende lernend oder lachend, oft genug auch beides gleichzeitig, während sechs Jahren Studium.

Meinen Eltern möchte ich für die Selbstverständlichkeit und Hingabe danken, mit der Sie mich unterstützt haben, seit ich denken kann. Für Ihren Rückhalt, Ihr Verständnis und Ihre Liebe.

Am meisten danke ich meiner Ehefrau Katrin für ihre Liebe und ihre Hilfe in dieser langen, oft stressigen Zeit. Für ungezähltes Probelesen, Korrigieren und dafür, dass sie mich mehrfach davon abgehalten hat, den Computer mitsamt dem Worddokument aus dem Fenster zu werfen.

Ebenso danke ich meinem Sohn Jonah, der zumindest in der Endphase dieser Doktorarbeit zeitweilig auf mich verzichten musste und mich dennoch stets freudig wiedererkannt hat. 


\section{Lebenslauf}

\section{Daniel Lankers}

Geburtsdatum: 1. Februar 1979

Geburtsort: Bonn-Duisdorf

Familienstand: verheiratet, 1 Kind

\section{Ausbildung}

1989 - 1998 Ernst-Moritz-Arndt-Gymnasium in Bonn, Abitur (2,4)

1998 - 1999 Bundeswehr, Grundwehrdienst

1999 - 2002 Ausbildung zum Krankenpfleger im GKH Bonn, Examen $(2,3)$

2002 - 2008 Studium der Humanmedizin, Ruhr-Universität Bochum $(1,5)$

\section{Praktisches Jahr}

1. Tertial: Innere Medizin, Prof. Weber und Prof. Hasselmann, Hôpital Civil, Straßburg, Frankreich

2. Tertial: Pädiatrie, Prof. Rieger, St. Josefs-Kinderklinik, Bochum

3. Tertial: Chirurgie, Prof. Eickhoff und Prof. Kemen, EVK Herne

\section{Berufliche Tätigkeiten}

2003 - 2008 Lehrkraft für Anatomie, Physiologie, Krankheitslehre und Altenpflege im Evangelischen Altenkrankenheim Essen

2004 - 2005 Ausbilder für Erste Hilfe und Lebensrettende Sofortmaßnahmen, Vitaguard Rettungsschule Bochum

Seit 01/2009 Assistenzarzt der Klinik für Anästhesiologie, Intensivmedizin und Schmerztherapie, Knappschaftskrankenhaus BochumLangendreer

\section{Publikationen}

2008 Kissler, S., Hötte, S.D., Lankers, D., Juckel, G., Schröder, S.G. (2008). Impact of vascular pathology on survival times of 173 dementia patients--Hachinski's ischemic score as a predictive tool for clinical purposes. Z Gerontol Geriatr. 41(1), 51-5.

2010 Hötte, S.D., Lankers, D., Kissler, S., Freyberger, H.J., Schröder, S.G. (2010). MMSE for survival prognostics in dementia. Psychiatr Prax 37(2), 78-83. Epub 2010 Feb 24. German. PMID: 20183772

Lankers, D., Kissler, S., Hötte, S.D., Freyberger, H.J., Schröder, S.G. (2010). Do dementia patients living at home live longer than in a nursing home? Z Gerontol Geriatr. 18. [Epub ahead of print] German.

PMID: 20237932

\section{Ehrenamtliches Engagement}

2002 - 2008 Mitglied der Fachschaft Medizin Bochum

2003 - 2005 Vorstandsmitglied und Finanzreferent der Fachtagung Medizin e.V.

2005 - 2007 Mitglied der Bundesvertretung der Medizinstudierenden

Deutschland e.V. (BVMD)

2006 - 2007 Studentischer Vertreter (Klinik) im Fakultätsrat 
Z Gerontol Geriat 2010 DOI 10.1007/s00391-010-0096-7 Eingegangen: 17. Januar 2008 Angenommen: 4. Januar 2010 (c) Springer-Verlag 2010

D. Lankers ${ }^{1}$. S. Kissler ${ }^{2}$ · S.D. Hötte ${ }^{3}$ · H.J. Freyberger ${ }^{4}$ · S.G. Schröder ${ }^{5}$

${ }^{1}$ Klinik für Anästhesiologie, Intensivmedizin und Schmerztherapie, Knappschaftskrankenhaus Bochum-Langendreer, Klinikum der Ruhr-Universität Bochum, Bochum

${ }^{2}$ Abteilung für Neurologie, Evangelisches Krankenhaus Lippstadt, Lippstadt

${ }^{3}$ Klinik für Anästhesiologie, operative Intensivmedizin, Schmerz- und Palliativmedizin, Marienhospital Herne, Klinikum der Ruhr-Universität Bochum, Herne

${ }^{4}$ Klinik und Poliklinik für Psychiatrie und Psychotherapie der Ernst-Moritz-Arndt-Universität Greifswald, Hanse-Klinikum Stralsund, Stralsund

${ }^{5}$ Arbeitsgruppe Gerontopsychiatrie der Ernst-Moritz-Arndt-Universität Greifswald, Klinik für Psychiatrie und Psychotherapie (mit Geriatrie), KMG Klinikum Güstrow, Güstrow

\section{Leben Demenzkranke zu Hause länger als im Heim?}

cherung (bis zu 54,5\% der Kosten), andererseits von der versorgten Person oder deren Angehörigen [6]. Zur Betreuung gab es im Jahr 2005 in Deutschland 9105 vollstationäre Alteneinrichtungen mit insgesamt ca. 749.00o Plätzen[13]. Etwa 60\% der Demenzkranken in Deutschland leben in Privathaushalten und werden $\mathrm{zu}$ rund $92 \%$ von Familienangehörigen versorgt [3]. Entsprechend muss ein großer Teil der Leistungen von den Angehörigen getragen werden. Hierzu zählen nicht nur die indirekten Kosten (z. B. geleistete Betreuungszeit), auch die Zuzahlung zu Medikamenten oder der Eigenanteil, der für die Unterkunft/Verpflegung des Erkrankten aufzubringen ist, bedeutet für die betroffenen Angehörigen eine große Belastung [10]. Auch wird sich in den kommenden Jahren die Versorgung Demenzkranker durch Angehörige zunehmend schwierig gestalten: Über 60\% der Personen, die als Hauptpflegende Betreuung in häuslichen Pflegearrangements übernehmen, sind über 55 Jahre alt und können in Zukunft die Pflegeverantwortung nicht mehr selbstverständlich übernehmen, da sie dann $u$. U. selbst nicht mehr in der Lage sind, körperlich belastende Pflegetätigkeiten zu leisten. Zudem steigt bei Frauen, die mit 8o\% den Großteil der Pflegenden darstellen, die Zahl der Berufstätigen [21].
Die Altenpflege steht damit vor einer Herausforderung: Wachsende Betreuungsaufgaben werden bei verminderten familiären Ressourcen zu bewältigen sein. In diesem Zusammenhang hat die Überlebenszeit von Demenzkranken - gerade auch für gesundheitspolitische Planungen - eine zentrale Bedeutung. Nach überwiegender Meinung beträgt die mittlere Überlebenszeit bei Altersdemenz, von den ersten klinischen Symptomen an gerechnet, etwa zehn Jahre [1, 2, 9]. Da psychosoziale Einflussfaktoren auf die Mortalität bei Demenz bislang noch zu wenig erforscht sind, wurde für die vorliegende Studie der Einfluss der Wohnform auf die Überlebenszeit von 173 Patienten untersucht. Gibt es diesbezüglich Unterschiede zwischen der häuslichen Pflege und der Unterbringung im Seniorenheim?

\section{Patienten und Methode}

In die Studie wurden Patienten mit den Diagnosen Alzheimer-Demenz (AD) und vaskuläre Demenz (VD) einbezogen. Als Einschlusskriterium galten dementsprechend die ICD-10-Nummern Foo und Fo1. Im Zeitraum zwischen 1995 und 2001 hatten 173 Demenzkranke mit AD und VD die Institutsambulanz des Westfälischen Zentrums Bochum (Universitätsklinik) zur weiteren Diagnostik aufge- unterschiedlichen Quellen finanziert: einerseits durch die Mittel der Pflegeversi- 


\begin{tabular}{|c|c|c|c|c|c|c|c|c|c|}
\hline & & \multirow{2}{*}{\multicolumn{2}{|c|}{$\begin{array}{l}\text { Gesamt- } \\
\text { kohorte }\end{array}$}} & \multirow{2}{*}{\multicolumn{2}{|c|}{$\begin{array}{l}\text { Datenlage } \\
\text { unvollständig }\end{array}$}} & \multirow{2}{*}{\multicolumn{2}{|c|}{$\begin{array}{l}\text { Datenlage } \\
\text { vollständig }\end{array}$}} & \multicolumn{2}{|l|}{ Wohnform } \\
\hline & & & & & & & & Seniorenheim & ZuHause \\
\hline \multicolumn{2}{|l|}{ Anzahl (n) } & \multicolumn{2}{|l|}{173} & \multicolumn{2}{|l|}{$28^{a}$} & \multicolumn{2}{|l|}{145} & 48 & 97 \\
\hline \multirow[t]{3}{*}{ MMST } & Med & \multicolumn{2}{|l|}{16} & \multicolumn{2}{|l|}{17,5} & \multicolumn{2}{|l|}{16} & 12,0 & 17 \\
\hline & M & \multicolumn{2}{|c|}{15,1} & \multicolumn{2}{|l|}{16,6} & \multicolumn{2}{|l|}{14,9} & $12,1^{b}$ & $16,3^{b}$ \\
\hline & SD & \multicolumn{2}{|l|}{7,0} & \multicolumn{2}{|l|}{8,5} & \multicolumn{2}{|l|}{6,7} & 6,5 & 6,5 \\
\hline \multirow{3}{*}{$\begin{array}{l}\text { Alter bei } \\
\text { Erstkontakt } \\
\text { (in Jahren) }\end{array}$} & Med & \multicolumn{2}{|c|}{79,2} & \multicolumn{2}{|l|}{81,7} & \multicolumn{2}{|l|}{78,9} & 84,3 & 76,7 \\
\hline & M & \multicolumn{2}{|c|}{78,8} & \multicolumn{2}{|l|}{80,3} & \multicolumn{2}{|l|}{78,5} & $83,6^{b}$ & $76,0^{\mathrm{b}}$ \\
\hline & SD & \multicolumn{2}{|l|}{8,9} & \multicolumn{2}{|l|}{8,5} & 8,9 & & 6,1 & 9,1 \\
\hline $\begin{array}{l}\text { Geschlechte } \\
\text { lung }(\mathrm{m} / \mathrm{w} \text { ir }\end{array}$ & $\begin{array}{l}\text { vertei- } \\
\%)\end{array}$ & 26 & 74 & 21 & 79 & 27 & 73 & $87^{b}$ & $66^{\mathrm{b}}$ \\
\hline $\begin{array}{l}\text { Wohnformv } \\
\text { lung (Sh/zH }\end{array}$ & $\begin{array}{l}\text { rtei- } \\
n \%)\end{array}$ & 32 & 68 & 25 & 75 & 33 & 67 & 33 & 67 \\
\hline
\end{tabular}

\begin{tabular}{|c|c|c|c|c|c|}
\hline & \multirow{2}{*}{$\begin{array}{l}\text { Gesamt- } \\
\text { kohorte }\end{array}$} & \multirow{2}{*}{$\begin{array}{l}\text { Datenlage } \\
\text { unvollständig }\end{array}$} & \multirow{2}{*}{$\begin{array}{l}\text { Datenlage } \\
\text { vollständig }\end{array}$} & \multicolumn{2}{|l|}{ Wohnform } \\
\hline & & & & Seniorenheim & Zu Hause \\
\hline Anzahl (n) & 173 & $28^{\mathrm{a}}$ & 145 & 48 & 97 \\
\hline $\begin{array}{l}\text { Mediane Überlebens- } \\
\text { zeit (in Monaten) }\end{array}$ & 44,0 & $6,7^{b}$ & 44,2 & $29,2^{c}$ & $55,5^{c}$ \\
\hline
\end{tabular}

\begin{tabular}{|c|c|c|c|c|}
\hline & \multirow[t]{2}{*}{ Signifikanz } & \multirow[t]{2}{*}{ Exp(B) (Hazard Ratio) } & \multicolumn{2}{|c|}{ 95,0\%-Konfidenzintervall für $\operatorname{Exp(B)}$} \\
\hline & & & Untere Grenze & Obere Grenze \\
\hline Alter & 0,000 & 1,046 & 1,023 & 1,070 \\
\hline Geschlecht & 0,000 & 2,583 & 1,678 & 3,978 \\
\hline MMST & 0,051 & 0,971 & 0,942 & 1,000 \\
\hline Diagnose & 0,212 & 0,754 & 0,484 & 1,174 \\
\hline \multicolumn{5}{|c|}{ MMST Mini-Mental-Status-Test } \\
\hline
\end{tabular}

sucht. Bei allen Patienten wurde bei der Erstuntersuchung der Mini-Mental-Status-Test (MMST) durchgeführt, außerdem wurden soziale Faktoren, wie die Wohnform, zum Zeitpunkt des Erstkontaktes festgehalten. Im Rahmen des Follow-up wurde der Überlebensstatus der Patienten telefonisch ermittelt. Im Mittel erfolgte diese Nachfrage 7,98 Jahre nach Erstkontakt. Bei bereits verstorbenen Personen wurde die Überlebenszeit nach Erstkontakt berechnet (• Tab. 1).

Bei 24 Patienten konnte das Überleben nach Erstvorstellung nicht ermittelt werden. Bei 4 weiteren Patienten war kein MMST durchgeführt worden, sodass in die Berechnung der Überlebenszeiten 145 Personen eingingen. Signifikante Unterschiede zwischen den Gruppen wurden für den MMST und das Alter bei Erstvorstellung ( $t$-Test für die Mittelwertgleichheit), die Überlebenszeit nach Erstvorstellung (Log-Rang-Test) und die Geschlechterverteilung ( $\chi^{2}$-Test nach Pearson) bestimmt. Die Patientendaten wurden nach den Wohnformen „im Seniorenheim lebend“ und „zu Hause lebend“ ausgewertet.

Für alle statistischen Verfahren wurde ein p-Wert <0,05 als statistisch signifikant festgelegt. Die statistische Bearbeitung der Daten erfolgte mit dem Programm SPSS 15.0 für Windows.

Die Analyse des relativen Sterberisikos wurde anhand des Cox-Regressionsmodells [5] unter Berücksichtigung der Kofaktoren Alter, Geschlecht, MMST und Diagnose (AD vs. VD) durchgeführt. Der
Bestimmung der medianen Überlebenszeit in Abhängigkeit von der Wohnform diente die Überlebenszeitanalyse nach Kaplan-Meier.

\section{Ergebnis}

Zwischen der Gesamtkohorte und der Gruppe von Personen mit unvollständiger Datenlage ergaben sich weder nach den uns vorliegenden soziodemographischen und klinischen Variablen noch nach den statistischen Auswertungen signifikante Unterschiede. Eine Ausnahme bildete die mediane Überlebenszeit nach Erstvorstellung, da der eruierbare Wert in der Gruppe der Patienten mit unvollständiger Datenlage auf lediglich 4 von $28 \mathrm{~Pa}$ tienten beruhte.

Zum Zeitpunkt der Datenerfassung (Juni 2006) waren 117 der 145 Patienten mit vollständiger Datenlage verstorben. Die 48 im Seniorenheim lebenden Patienten waren bei Erstkontakt etwa 7,1 Jahre älter als die 97 häuslich Gepflegten $(\mathrm{p}<\mathrm{0}, 001)$. Das durchschnittliche Ergebnis des MMST war um 4,2 Punkte niedriger als das der zu Hause Lebenden ( $\mathrm{p}<\mathrm{o}, 001$; - Abb. 1,). Im Seniorenheim lebten 42 Frauen (87,5\%), zu Hause $64(66 \%), p=0,006$.

Die Berechnungen nach Kaplan-Meier ergaben eine mediane Überlebenszeit der Patienten im Seniorenheim von 29,2 Monaten (2,43 Jahre) im Gegensatz zur Überlebenszeit der zu Hause Lebenden mit 55,5 Monaten (4,63 Jahre; $\mathrm{p}=0,043$;

\section{- Abb. 2, - Tab. 2).}

Die Adjustierung des Cox-Regressionsmodells an den Variablen MMSTWert, Alter, Geschlecht und Diagnose (AD vs. VD) erbrachte die in $\bullet$ Tab. 3 dargestellten Werte.

Die Berechnung der Hazard Ratio nach Cox im Vergleich zwischen den Wohnformen ergab den Wert 1,531 (95\%-Konfidenzintervall: 1,005-2,333), $\mathrm{p}=0,047$. Aus diesem Ergebnis lässt sich ablesen, dass das relative Sterberisiko von Demenzpatienten im Seniorenheim - adjustiert an den oben genannten Variablen - um 53,1\% erhöht ist. 


\section{Diskussion}

Unsere Untersuchung zeigt, dass ein deutlicher Unterschied in der Überlebenszeit an Demenz Erkrankter besteht, je nachdem, ob sie im Seniorenheim oder zu Hause gepflegt wurden. Zu Hause versorgte Demenzpatienten haben nach unserer Studie ein um 53,1\% niedrigeres relatives Sterberisiko als die Patienten im Seniorenheim. Einerseits ergeben die Berechnungen nach Kaplan-Meier bei der Gruppe der zu Hause lebenden Patienten ein um 26,3 Monate (2,2 Jahre) längeres medianes Überleben. Andererseits bleibt bei dieser Betrachtung unberücksichtigt, dass die Gruppe der im Seniorenheim Lebenden bereits zu Anfang des Betrachtungszeitraumes ein um 7,1 Jahre höheres Alter und, dem um durchschnittlich 4,2 Punkte schlechteren MMST-Wert entsprechend, eine niedrigere kognitive Fähigkeit durch die im Mittel schon weiter fortgeschrittene Demenz aufweist. Berücksichtigt man diesen Sachverhalt ebenso wie die unterschiedliche Geschlechtsverteilung, ergibt sich in der Regressionsberechnung nach dem Cox-Modell (Hazard Ratio) die niedrigere Sterbewahrscheinlichkeit der häuslich Versorgten. Dies ließe in einer prospektiv angelegten Studie mit einheitlichen Patientengruppen eine deutlich höhere Überlebenszeit für zu Hause versorgte Patienten erwarten.

Der erhebliche Unterschied der Überlebenszeiten zwischen den Patienten mit vollständiger und unvollständiger $\mathrm{Da}$ tenlage lässt sich nach einer Reevaluation der Einzelfälle darauf zurückführen, dass in der kleinen Gruppe der Patienten $(n=4)$, bei denen keine psychometrische Untersuchung durchgeführt, wohl aber die Überlebenszeit bestimmt wurde, bereits ein sehr fortgeschrittenes Demenzstadium vorlag. Ein - wenn auch geringer - Bias könnte auf dieser Selektion beruhen. Die ermittelten medianen Überlebenszeiten müssten somit allenfalls geringfügig nach unten korrigiert werden.

In dieser Studie wird zwischen Alzheimer- und vaskulärer Demenz unterschieden, obwohl neuere Untersuchungen eine große Überschneidung dieser beiden nosologischen Entitäten auf histopathologischer [18] und klinischer [22] Ebene zeigen. Der Einfluss der Demenzätiolo-

Z Gerontol Geriat $2010 \quad$ DOI 10.1007/s00391-010-0096-7

(c) Springer-Verlag 2010

D. Lankers · S. Kissler · S.D. Hötte · H.J. Freyberger · S.G. Schröder Leben Demenzkranke zu Hause länger als im Heim?

\section{Zusammenfassung}

Wie eine Vielzahl von Studien zeigt, nimmt die Zahl der Neuerkrankungen an Demenz in unserer Gesellschaft jährlich zu. In diesem Zusammenhang stehen zwei gesellschaftliche Aufgaben im Vordergrund: die Sicherstellung der adäquaten Betreuung von an Demenz Erkrankten auch in Zukunft sowie die Deckung der Kosten, die dadurch verursacht werden. Dabei hat die Überlebenszeit von Demenzkranken - gerade auch für gesundheitspolitische Planungen - eine zentrale Bedeutung Deshalb lautet die Fragestellung unserer über 8 Jahre angelegten Follow-up-Studie: Beeinflusst die Wohnform die Überlebenszeit Demenzkranker? Von 173 Patienten mit Alzheimer- oder vaskulärer Demenz (ICD-10-Nr. F00 und F01) wurden klinische und soziale Daten erfasst. Bei verstorbenen Patienten wurde nach Befragung der Angehörigen das exakte Todesdatum ermittelt. Anschließend wurden die Überlebenszeiten mit der entsprechenden Wohnform korreliert. Unter Anwendung des Cox-Modells wurden Sterberisiken ermittelt. Die Untersuchung zeigt, dass ein deutlicher Unterschied in der Überlebens zeit Demenzkranker besteht, je nachdem, ob sie im Seniorenheim oder zu Hause gepflegt wurden. Adjustiert nach Alter und kognitiver Leistungsminderung hatten Patienten im Seniorenheim ein um $53,1 \%$ höheres relatives Sterberisiko (Hazard Ratio) als zu Hause Betreute $(p=0,047)$. Weiterführende prospektive Studien zur Überlebenszeit in Abhängigkeit von der Wohnform sollten durchgeführt werden.

Schlüsselwörter Wohnform · Demenz · Überlebenszeit . Seniorenheim · Ambulante Pflege

\section{Do dementia patients living at home live longer than in a nursing home?}

\section{Abstract}

Many studies have shown that the number of new dementia diagnoses in Germany is increasing yearly. Thus, two social tasks are important: the adequate support and care of dementia patients, now and in the future, as well as covering the costs thereof. The survival period of dementia patients has a central meaning - especially for health policy planning. Therefore, the question of our 8-year follow-up study was whether living conditions affect the survival period of dementia patients? A total of 173 dementia outpatients (ICD-10 numbers F00 and F01) were screened for survival time and living conditions. For deceased patients, a close reference person was interviewed, and the exact date of death was recorded. For statistical evaluation, the Cox proportional hazard model was used and dying risks were determined. Our investigation shows that a clear difference exists in the survival period of dementia patients, according to whether they have lived at home or in a senior citizen's home. Patients in senior citizen's homes had a higher relative dying risk of around $53.1 \%$ (hazard ratio), than for those cared for at home $(p=0.047)$. Prospective research is needed to gain more evidence about the impact of social factors, e.g. living conditions, on the survival time of demented patients.

\section{Keywords}

Living conditions · Dementia · Survival .

Home care $\cdot$ Nursing home 

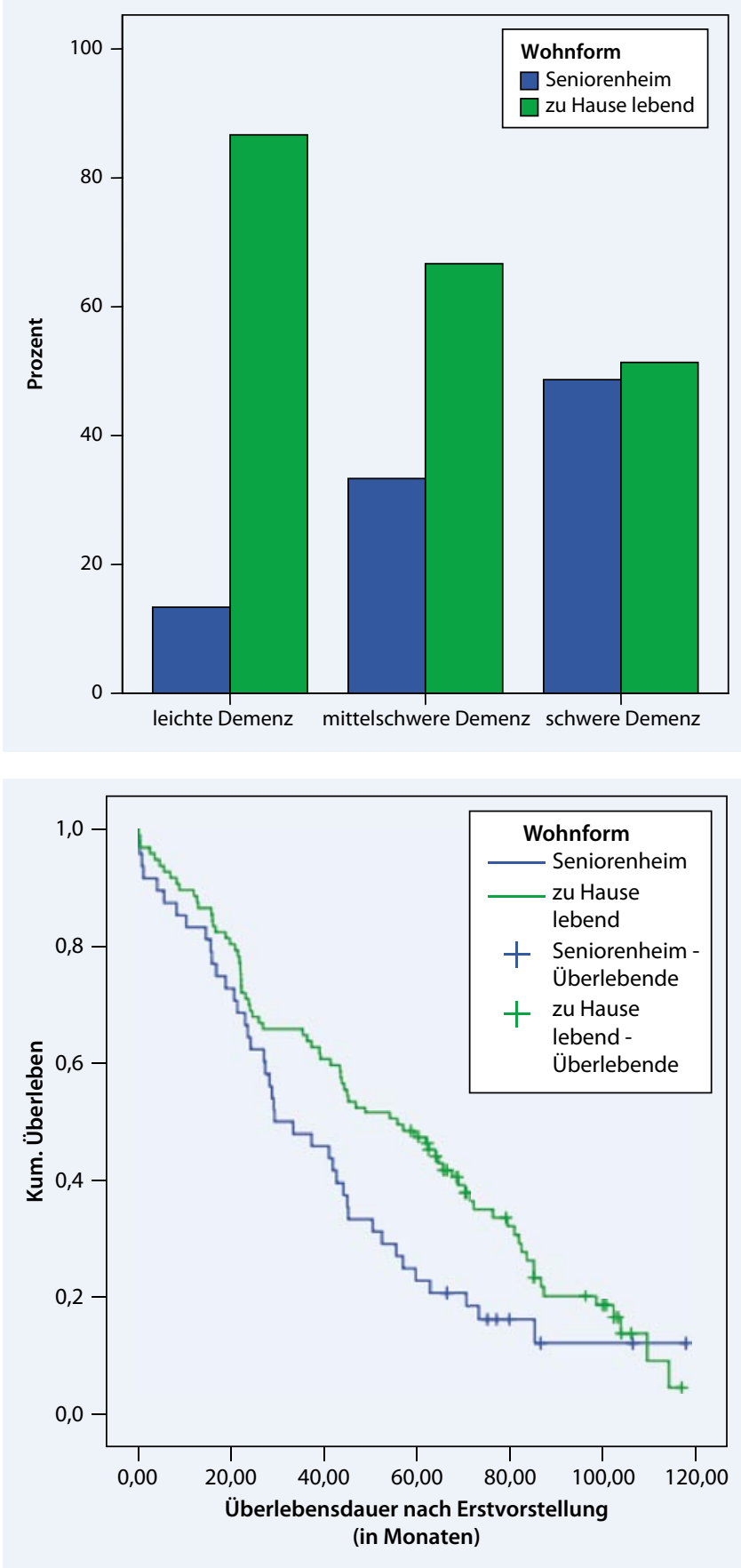

Abb. $1<$ Schweregrad der Demenz nach Wohnform (gemäß MMST $\leq 20$ bzw. $\leq 10$ )

Abb. $2<$ Überlebenszeit nach Wohnform

gie auf die Sterblichkeit wird uneinheitlich bewertet: Je nach Studie hat die AD eine etwas günstigere Prognose als die VD [16] oder beide Hauptdemenzformen unterscheiden sich nicht wesentlich bezüglich der Überlebenszeit [9].

Die unterschiedliche Geschlechterverteilung wurde zwar in der Cox-Regression berücksichtigt - so zeigen Frauen unserer Studie zufolge eine ca. 2,5-fach erhöhte Überlebenswahrscheinlichkeit gegenüber Männern -, allerdings wird auch der Einfluss des Geschlechts auf die Sterblichkeit von Demenzkranken kontrovers beurteilt, ohne dass es diesbezüglich einen wissenschaftlichen Konsens gibt [9].

Aufgrund des retrospektiven Designs der Studie konnten keine Nebendiagnosen erfasst werden. Auch wenn es Hinweise darauf gibt, dass die Mortalität der Erkrankten hauptsächlich vom Alter und Schweregrad der Demenz und weniger von weiteren gravierenden Erkrankungen (wie z. B. Herz-Kreislauf- oder Krebserkrankungen) abhängt [2], sollten diese somatischen Diagnosen in künftigen Studi- en erfasst werden. Das gilt auch für weitere wichtige Einflussfaktoren wie die Medikation (z. B. bezüglich Sturzgefährdung) oder die Art der Betreuung der zu Hause versorgten Patienten (durch Lebenspartner, Kindergeneration, ambulanten Pflegedienst).

Die Abschätzung der Überlebenszeiten von Demenzkranken hat unseres Erachtens u. a. die praktische Bedeutung, Angehörige bezüglich der Prognose fundierter beraten zu können, damit diese besser ihre eigenen Grenzen erkennen und sich entsprechend für eine häusliche oder institutionelle pflegerische Versorgung entscheiden können [12]. Dies hätte im Sinne einer psychiatrischen Primärprävention eine Verbesserung des Gesundheitszustandes der pflegenden Angehörigen zur Folge, da diese eine Hochrisikopopulation für psychiatrische Morbidität darstellen. Studien zur häuslichen Pflege von Demenzkranken konstatieren eine signifikant höhere Belastung der pflegenden Angehörigen im Vergleich zu Pflegenden nicht an Demenz Leidender [4]. Ihren eigenen Angaben zufolge sind 42\% der Angehörigen schwer, $41 \%$ sogar sehr schwer belastet [14]. Angehörige, die sich intensiv um einen Pflegebedürftigen kümmerten, litten häufiger an körperlichen Beschwerden wie Erschöpfung, Gliederschmerzen, Herz- und Magenproblemen als die Normalbevölkerung [8].

Unter anderem aus diesen Gründen gilt Demenz als Hauptrisikofaktor für eine Heimunterbringung [23]. Durch die professionelle Versorgung kann im Seniorenheim besonders auf somatische Probleme meist besser eingegangen werden [11]. So stellen Mangelernährung und Dehydrierung zentrale Probleme der Pflege von Demenzkranken dar [20], denen im häuslichen Milieu häufig schwerer zu begegnen ist. Der fünfte Altenbericht der Bundesregierung kommt zu dem Ergebnis, dass bei pflegenden Angehörigen das Wissen um die Bedürfnisse der an Demenz leidenden Personen oft nicht ausreichend ist [3]. Auch gibt es Hinweise dafür, dass dem gestörten Schlaf-WachRhythmus von Demenzkranken, der zu Hause eine große Belastung für die Angehörigen darstellt, im Altenheim durch den Schichtdienst besser begegnet werden kann [7]. Für die Heimunterbrin- 
gung spricht auch, dass nach Jakubaschk die Selbstgefährdung der an Demenz Erkrankten zu Hause höher einzuschätzen ist als im Pflegeheim [15] und Seniorenheime häufig baulich besser an die Anforderungen Pflegebedürftiger angepasst sind [13]. Dementgegen gibt es Hinweise, dass ein inadäquater Einsatz von Psychopharmaka in Seniorenheimen in Deutschland häufig ist, bei einer Verordnungsrate von $55,6 \%$ der Bewohner [19]. Inwieweit sich dies auf die Überlebenszeit Demenzkranker auswirkt, bleibt zu untersuchen. Auch das Thema Lebensqualität hat noch wenig Beachtung erlangt, wobei vermutlich häufig in Pflegeheimen die sozialen Bedürfnisse der Kranken (u. a. zwischenmenschlicher Kontakt und sinnvolle Beschäftigung) weniger befriedigt werden $[11,19]$.

Nach unserer Erkenntnis wurden bislang nur wenige Studien zur Frage der Versorgungsqualität von Patienten mit Demenz in Abhängigkeit von der gewählten Wohnform durchgeführt. Keine davon untersuchte den Einfluss der Wohnform auf die Mortalität. Unsere Ergebnisse zeigen jedoch gerade hier signifikante Unterschiede und weisen auf einen noch zu wenig beachteten Bereich der Versorgungsforschung hin.

\section{Fazit}

\section{- Die Mortalität von Alzheimer-Pati- enten hängt hauptsächlich vom Alter und Schweregrad der Demenz ab. \\ - Zu Hause gepflegte Patienten zei- gen unter Alters-, Geschlechts- und Schweregradadjustierung ein gerin- geres relatives Sterberisiko (Hazard Ratio) als im Seniorenheim Betreute. \\ - Demenzkranke Frauen leben länger als demenzkranke Männer. \\ - In der Versorgungsforschung wird der Wohnform von Demenzkranken, trotz eines erheblichen Einflusses auf die Überlebenszeit, bislang zu wenig Be- achtung geschenkt.}

\section{Korrespondenzadresse \\ PD Dr. S.G. Schröder}

Arbeitsgruppe Gerontopsychiatrie der ErnstMoritz-Arndt-Universität Greifswald , Klinik für Psychiatrie und Psychotherapie (mit Geriatrie), KMG Klinikum Güstrow,

Friedrich-Trendelenburg-Allee 1, 18273 Güstrow s.schroeder@kmg.ag

Interessenkonflikt. Der korrespondierende Autor hat Referentenhonorare und Kongressunterstützungen von folgenden Pharmafirmen erhalten: AstraZeneca, Bristol-Myers Squibb, Dr. Köhler Chemie Eisai, Janssen-Cilag, Merz, Novartis, Pfizer und Servier.

\section{Literatur}

1. Bickel H (2000) Demenzsyndrom und Alzheimer Krankheit: Eine Schätzung des Krankenbestandes und der jährlichen Neuerkrankungen in Deutschland. Gesundheitswesen 62:211-218

2. Bonsignore M, Heun R (2003) Mortality in Alzheimer's disease. Dement Geriatr Cogn Disord $15: 231-236$

3. Bundesministerium für Familie, Senioren, Frauen und Jugend (2005) Fünfter Bericht zur Lage der älteren Generation. Eigenverlag, Berlin

4. Bundesministerium für Familie, Senioren, Frauen und Jugend (2002) Vierter Bericht zur Lage der älteren Generation. Eigenverlag, Berlin

5. Cox D (1972) Regression models and life tables. J R Stat Soc B 34:187-220

6. Eurofamcare (2004) National Background report for Germany 2004. http://www.uke.uni-hamburg. de/eurofamcare

7. Gehrman P, Marler M, Martin JL et al (2004) The timing of activity rhythms in patients with dementia is related to survival. J Gerontol A Biol Sci Med Sci 59:1050-1055

8. Gräßel E (1998) Häusliche Pflege demenziell und nicht-demenziell Erkrankter. Teil II: Gesundheit und Belastung der Pflegenden. Z Gerontol Geriatr 31:57-62

9. Guehne U, Riedel-Heller S, Angermeyer MC (2005) Mortality in dementia. Neuroepidemiology 25:153-162

10. Hallauer JF, Berger K, Ruckdäschel S (2002) Sozio-ökonomische Aspekte - nationale und internationale Untersuchungsergebnisse. In: Hallauer JF, Kurz A (Hrsg) Weißbuch Demenz - Versorgungssituation relevanter Demenzerkrankungen in Deutschland. Thieme, Stuttgart, S 19-23

11. Hancock GA, Woods B, Challis D, Orrell M (2006) The needs of older people with dementia in residential care. Int J Geriatr Psychiatry 21:43-49

12. Hötte SD, Lankers D, Kissler S et al (2010) MMST zur Überlebensdiagnostik bei Demenz. Psychiat Prax 37 (in Druck)

13. Infratest Sozialforschung (2003) Hilfe- und Pflegebedürftige in Privathaushalten in Deutschland 2002. Schnellbericht. http://www.tns-infratest-sofo.com/downloads/mug3sb.pdf

14. Infratest Sozialforschung (2006) Hilfe- und Pflegebedürftige in Alteneinrichtungen in Deutschland 2005. Schnellbericht. http://www.bmfsfj.de/ RedaktionBMFSFJ/Abteilung3/Pdf-Anlagen/hilfeund-pflegebeduerftige-in-alteneinrichtungen

15. Jakubaschk J, Hunziker R (1988) Patienten mit se niler Demenz - eine Gruppe der neuen Langzeitpatienten. Psychiatr Prax 15:115-121
16. Kissler S, Hötte SD, Lankers D et al (2008) Welchen Einfluss haben zerebrale Durchblutungsstörungen auf die Überlebenszeit Demenzerkrankter? Z Gerontol Geriatr 41:51-55

17. Knesebeck $\mathrm{O}$ vd, Döhner $\mathrm{H}$, Kaduszkiewicz $\mathrm{H}$ et al (2006) Forschung zur Versorgung im höheren Lebensalter. Prävention, Case Management und Versorgung von Demenz. Bundesgesundheitsbl Gesundheitsforsch Gesundheitsschutz 49:167-174

18. MRC CFAS (2001) Pathological correlates of lateonset dementia in a multicentre, community-based population in England and Wales. Lancet 357:169-175

19. Pantel J, Weber B, Bockenheimer-Lucius $G$, Ebsen I (2007) Psychopharmaka im Altenpflegeheim. http://www.bhf-bank-stiftung.de/2007/Abschlussbericht.pdf

20. Rainer MK, Mucke HA, Masching AJ et al (2005) Zeitgemäßes Management von nicht kognitiven Symptomen bei Demenz. Psychiatr Prax 32:31-38

21. Schneekloth U, Müller U (2000) Wirkungen der Pflegeversicherung. Schriftenreihe des Bundesministeriums für Gesundheit. Bd. 127. Nomos, Baden-Baden

22. Schröder SG (2006) Psychopathologie der Demenz. Symptomatologie und Verlauf dementieller Erkrankungen. Schattauer, Stuttgart

23. Weyerer S, Schaufele M, Schrag A, Zimber A (2004) Demenzielle Störungen, Verhaltensauffälligkeiten und Versorgung von Klienten in Einrichtungen der Altentagespflege im Vergleich mit Heimbewohnern: eine Querschnittsstudie in acht badischen Städten. Psychiatr Prax 31:339-345 